\title{
The changing face of venous thromboembolism management in England
}

\author{
Sreeram V Ramagopalan*,1, Robert Carroll ${ }^{1}$, Maria Ulvestad ${ }^{2}$, Faisal Mehmud ${ }^{3}$ \& Raza \\ Alikhan ${ }^{4}$ \\ ${ }^{1}$ Centre for Observational Research \& Data Sciences, Bristol-Myers Squibb, Uxbridge UB8 1DH, UK \\ ${ }^{2}$ Medical Department, Bristol-Myers Squibb, 1366 Lysaker, Norway \\ ${ }^{3}$ Medical Department, Bristol-Myers Squibb, Uxbridge UB8 1DH, UK \\ ${ }^{4}$ Haemophilia \& Thrombosis Centre, University Hospital Wales, Cardiff, UK \\ *Author for correspondence: Tel.: +44 189552 3000; sreeram.ramagopalan@bms.com
}

Aim: Venous thromboembolism (VTE), which comprises deep vein thrombosis and pulmonary embolism, poses a global disease burden. Vitamin $\mathrm{K}$ antagonists have traditionally been the mainstay of treatment; however, the non-vitamin $\mathrm{K}$ oral anticoagulants (NOACs) are emerging as an alternative. The relative use of these treatment classes in the real world is unknown. Patients \& methods: We performed a retrospective study using data from the UK Clinical Practice Research Datalink to understand VTE treatment patterns. Results: NOACs have unseated vitamin K antagonist as the main form of VTE patient treatment in England. Conclusion: The data highlight how comfortable physicians have become in using NOACs to treat VTE in England and it is likely that the increasing use of NOACs will continue.

First draft submitted: 11 February 2019; Accepted for publication: 6 March 2019; Published online: 25 March 2019

Keywords: Clinical Practice Research Datalink $\bullet$ database $\bullet$ deep vein thrombosis $\bullet$ England $\bullet$ epidemiology $\bullet$ hospital episode statistics $\bullet$ oral anticoagulant $\bullet$ pulmonary embolism • treatment $\bullet$ venous thromboembolism

The term venous thromboembolism (VTE) encompasses the presence of pulmonary embolism (PE) and/or deep vein thrombosis (DVT) [1]. It is estimated that VTE affects over 10 million people worldwide annually [1]. Anticoagulation therapy is the mainstay of treatment for VTE patients [1]. The use of oral vitamin K antagonists (VKAs), including warfarin, has historically been the commonest approach to anticoagulation treatment in VTE patients without cancer, but it is associated with a number of practical challenges, including numerous food and medication interactions, and the need for regular anticoagulation monitoring [2]. The non-vitamin K antagonist oral anticoagulants (NOACs) have emerged as viable alternatives to VKA therapy in patients with VTE [2]. Prospective randomized clinical trials and real-world evidence comparing NOACs to VKAs demonstrate comparable efficacy and the potential for improved safety, in terms of reductions in major bleeding such as intracranial haemorrhage (ICH) [3-5].

Data to understand how VKA or NOACs are currently being used to treat patients with VTE in routine clinical practice is, however, limited. We therefore conducted an observational, retrospective cohort study that evaluated VTE treatment in England using the Clinical Practice Research Datalink (CPRD) linked to Hospital Episode Statistics (HES) data [6]. This dataset links anonymized data from the medical records of patients registered with participating general practices in England (the CPRD dataset) with administrative records regarding their clinical care in NHS hospitals (the HES dataset) [6]. The dataset therefore includes longitudinal information on diagnoses, symptoms, laboratory tests and prescriptions issued by the GP in addition to information on referrals to specialists, hospital diagnoses, hospital procedures and deaths [6]. While the dataset does not capture private healthcare encounters, it covers approximately $4 \%$ of the English population and has been found to be representative of the UK population with regard to sex, age and ethnicity [6]. The data source has been used extensively in drug safety and health services research resulting in over 2000 peer-reviewed publications [6]. Patients registered with a general practice in the CPRD linked to HES who experienced a first ever VTE event during the cohort identification period (1 January 2013 to 1 July 2017) and were treated with apixaban, rivaroxaban or warfarin within 30 days of VTE

Future Medicine 


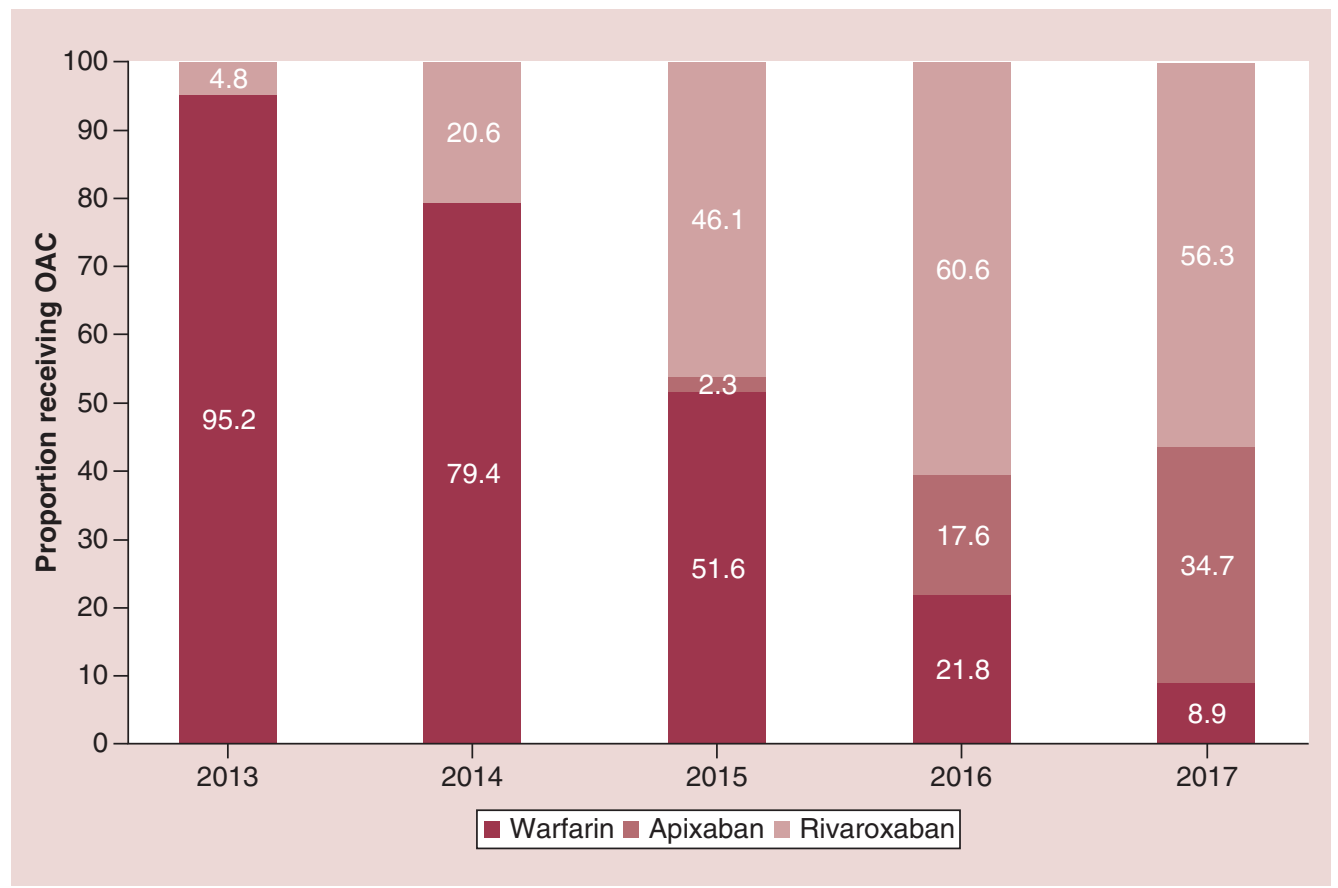

Figure 1. Proportion of first-time venous thromboembolism patients receiving each oral anticoagulant, by calendar year. Please note that the figure does not capture the full calendar year 2017, as data collection ended on 31 July 2017. Number of patients in each year were 960 (2013); 836 (2014); 740 (2015); 574 (2016) and 190 (2017).

OAC: Oral anticoagulant.

diagnosis were included. Patients were excluded if they were less than 18 years of age at index date; had a previous diagnosis of atrial fibrillation (AF), mechanical heart valve replacement or mitral stenosis, a previous prescription of any OAC at any time prior to the index date (to identify incident users only); or had a cancer diagnosis within 90 days of the index date. The study was approved by the CPRD's Independent Scientific Advisory Committee.

A total of 3300 VTE patients were identified (warfarin, $n=2102$; apixaban, $n=184$; rivaroxaban, $n=1014$ ); $34.9 \%$ experienced PE, 61.8\% DVT and 3.2\% both. Proportions of incident VTE patients receiving warfarin decreased over time - from $95.2 \%$ in 2013 to $8.9 \%$ in 2017 ; rivaroxaban use increased from $4.8 \%$ in 2013 to $56.3 \%$ in 2017 , and apixaban use increased from $0 \%$ in 2014 to $34.7 \%$ in 2017 (Figure 1).

These data clearly show NOACs unseating warfarin as the standard of care for VTE in 2016, with changes in the individual NOACs reflecting licensing and market availability. The attitudes and prescribing characteristics of the physician are significant factors in determining the uptake of new agents in clinical practice [7]. Individual physicians may be reluctant to prescribe NOACs in preference to VKA therapy due to their own long-standing history of VKA prescribing and the perceived concerns regarding safety and benefits of NOACs [7]. However, in this analysis, it is clear that physicians have become increasingly comfortable, over a short period of time, in using NOACs for the treatment of VTE without cancer. This is likely helped by NICE guidelines and robust clinical trial evidence [8]. This sea change in the management of DVT and PE may be beneficial to patients and the healthcare system alike. Potential benefits include reduced healthcare costs associated with fewer and shorter hospital stays, no routine coagulation monitoring appointments and a reduction in ICH [2]. Taken together, the potential benefits associated with the use of NOACs for VTE treatment can lead to significant improvements in the quality of life and outcomes of VTE patients. However limited data from real-world clinical practice are available to compare these outcomes and further research is needed [2].

\section{Conclusion}

In summary, we see here the significant change in management of VTE patients in England. Although clinical trial data support the use of NOACs as an effective and safe alternative to VKA therapy in VTE contexts, there is a need to consider the individual clinical profile and risk factors of patients prior to NOAC initiation. Nevertheless, 
given NOACs potential to improve the outcomes of patients with VTE, it is likely that the observed increase in the frequency of their use will continue.

\section{Future perspective}

As experience with the use of NOACs in VTE grows, so too will the amount of observational evidence available on the effectiveness and safety of these drugs. We will also have a better understanding of the impact of VTE treatment at the patient level (e.g., quality of life) and at the healthcare system level (healthcare resource use). Should this evidence suggest that the net benefit of NOACs in individuals with VTE is favorable, then the use of NOACs to treat VTE may rise significantly in the future.

\section{Summary points}

- Venous thromboembolism (VTE), which comprises deep vein thrombosis and pulmonary embolism, poses a significant global disease burden.

- Vitamin K antagonists (VKAs) have traditionally been the mainstay of treatment. However, VKA therapy is associated with challenges including the need for regular anticoagulation monitoring.

- The non-vitamin K oral anticoagulants (NOACs) are emerging as an alternative for VTE management.

- We show here using data from the UK Clinical Practice Research Datalink that NOACs have since 2016 unseated VKA as the main form of VTE patient treatment.

- The data highlights how comfortable physicians have become in using NOACs to treat VTE in England and it is likely that the increasing use of NOACs will continue.

\section{Acknowledgments}

Data analysis was performed by R Donaldson and D Lambrelli at Evidera.

Financial \& competing interests disclosure

This study was supported by Bristol Myers-Squibb and Pfizer. SV Ramagopalan, R Carroll, M Ulvestad and F Mehmud are employees of Bristol Myers-Squibb. R Alikhan has previously received consultancy fees from Bayer, Boehringer-Ingelheim, Bristol-Myers Squibb, Pfizer and Daiichi-Sankyo. The authors have no other relevant affiliations or financial involvement with any organization or entity with a financial interest in or financial conflict with the subject matter or materials discussed in the manuscript apart from those disclosed.

No writing assistance was utilized in the production of this manuscript.

\section{Open access}

This work is licensed under the Attribution-NonCommercial-NoDerivatives 4.0 Unported License. To view a copy of this license, visit http://creativecommons.org/licenses/by-nc-nd/4.0/

\section{References}

1. Di Nisio M, van Es N, Büller HR. Deep vein thrombosis and pulmonary embolism. Lancet 388(10063), 3060-3073 (2016).

2. Keeling D, Alikhan R. Management of venous thromboembolism - controversies and the future. Br. J. Haematol. 161(6), 755-763 (2013).

3. Dawwas GK, Brown J, Dietrich E, Park H. Effectiveness and safety of apixaban versus rivaroxaban for prevention of recurrent venous thromboembolism and adverse bleeding events in patients with venous thromboembolism: a retrospective population-based cohort analysis. Lancet Haematol. 6(1), e20-e28 (2019).

4. Agnelli G, Buller HR, Cohen A et al. Oral apixaban for the treatment of acute venous thromboembolism. N. Engl. J. Med. 369(9), 799-808 (2013).

5. EINSTEIN Investigators, Bauersachs R, Berkowitz SD et al. Oral rivaroxaban for symptomatic venous thromboembolism. N. Engl. J. Med. 363(26), 2499-2510 (2010).

6. Herrett E, Gallagher AM, Bhaskaran K et al. Data Resource Profile: Clinical Practice Research Datalink (CPRD). Int. J. Epidemiol. 44(3), 827-836 (2015).

7. Cohen AT, Lip GY, De Caterina R et al. State of play and future direction with NOACs: an expert consensus. Vascul. Pharmacol. 106, 9-21 (2018).

8. Stubbs MJ, Mouyis M, Thomas M. Deep vein thrombosis. BMJ 360, k351 (2018). 
Short Communication Ramagopalan, Carroll, Ulvestad, Mehmud \& Alikhan 